Original Research Paper

\title{
New Approach in Optimization Using Variant of the First Derivative (CFDSM)
}

\author{
${ }^{1}$ Tahseen Ali Jabbar, ${ }^{2}$ Saleh Ismael Najem and ${ }^{1}$ Sadoun Fahad Dakhil \\ ${ }^{1}$ Fuel and Energy, Technical College of Basrah, Southern Technical University, Basrah, Iraq \\ ${ }^{2}$ Mechanical Engineering, Engineering College, University of Basrah, Basrah, Iraq
}

\author{
Article history \\ Received: $13-07-2018$ \\ Revised: 04-02-2019 \\ Accepted: 19-12-2019 \\ Corresponding Author: \\ Tahseen Ali Jabbar \\ Fuel and Energy, Technical \\ College of Basrah, Southern \\ Technical University, Basrah, \\ Iraq \\ Email: tahseenali.ali114@gmail.com
}

\begin{abstract}
Optimization methods are considered very important in engineering and industrial fields. Many of these methods depend on regression function for finding optimal values. In this research, a new method was adopted based on the change of the signal of the first derivative, where the update is based on the reference of the first derivative of the function. The results show that this method can be used to find optimization, as well as that it is very sensitive to the chosen range.
\end{abstract}

Keywords: Optimization, Gradient, First Derivative

\section{Introduction}

Optimization is mathematical method that care to calculate of the extreme (minima and maxima) of numbers, functions, or systems. Optimization techniques are widely used in many parts of science and engineering such as in business and industry. Many of authors studied the optimization methods and write a book on it, from they, Rao (1996) wrote a book titled " Engineering Optimization Theory and Practice", where this book including the explanations for the types of optimization methods. Rustagi (2014) wrote a book under the title "Optimization Techniques in Statistics". Pardalos and Romeijn (2013) presented a book under the title "handbook of global optimization". Stephen and Vandenberghe (2004) presented a book "Convex Optimization". The researches, which has been interested in studying the radiation in combustion chamber, will be discussed with some conciseness.

The aim of the present work is to developed a new approach to find the optimum values for an objective functions.

\section{Change First Derivative Signal in Optimization}

The optimum value of objective function occurs when the first derivative equal to zero. Therefore many of optimization method depends on this idea.

Figure 1a show the optimum value of objective function occurs at $x^{*}=1$, where objective function as:

$$
y=(x-1)^{2}
$$

The function has negative gradient all range $x<1$ and positive gradient for all range $x>1$ due to the function has minimizing value in this range $\left(x^{*}=1\right.$ or $\left.\frac{d f}{d x}(1)=0\right)$. From this option, one can calculate the optimum value by reducing the range of the independent variable. Therefore the variant of the first derivative can be used in optimization methods. Using a variant of the first derivative method for optimization (Change first derivative signal method in optimization CFDSM) will be explained below.

In this method the average value of full range will be represented update the independent variable and can be writing as:

$$
X_{\text {new }}=\left(\frac{X_{\max }+X_{\min }}{2}\right)
$$

where, $X_{\max }$ and $X_{\min }$ represent a maximum and minimum value of the range for independent variable respectively.

The gradient at $X_{n e w}$ is taken one of the values (negative, zero and positive). If it has zero value that means optimum value, but for negative and positive, the update value become as in the Table 1:

Table 1: The update of independent variable

\begin{tabular}{ll}
\hline Gradient & Update \\
\hline Negative & $X_{\min }=X_{\text {new }}$ \\
Positive & $X_{\max }=X_{\text {new }}$ \\
\hline
\end{tabular}


That is mean for any next iteration the singe of first derivative must be negative and positive at $x_{\min }$ and $x_{\max }$ respectively where function has minimum value in this range. But for maximum value, the singe of first derivative must be positive and negative at $x_{\min }$ and $x_{\max }$ respectively. The above procedure will be repeated till the absolute value of gradient at update value of $x\left(x_{\text {new }}\right)$ become less than small value (for example less than $\left.1 \times 10^{-6}\right)$ as shown in Fig. 1b. The flow chart of this method can be shown in Fig. 2:

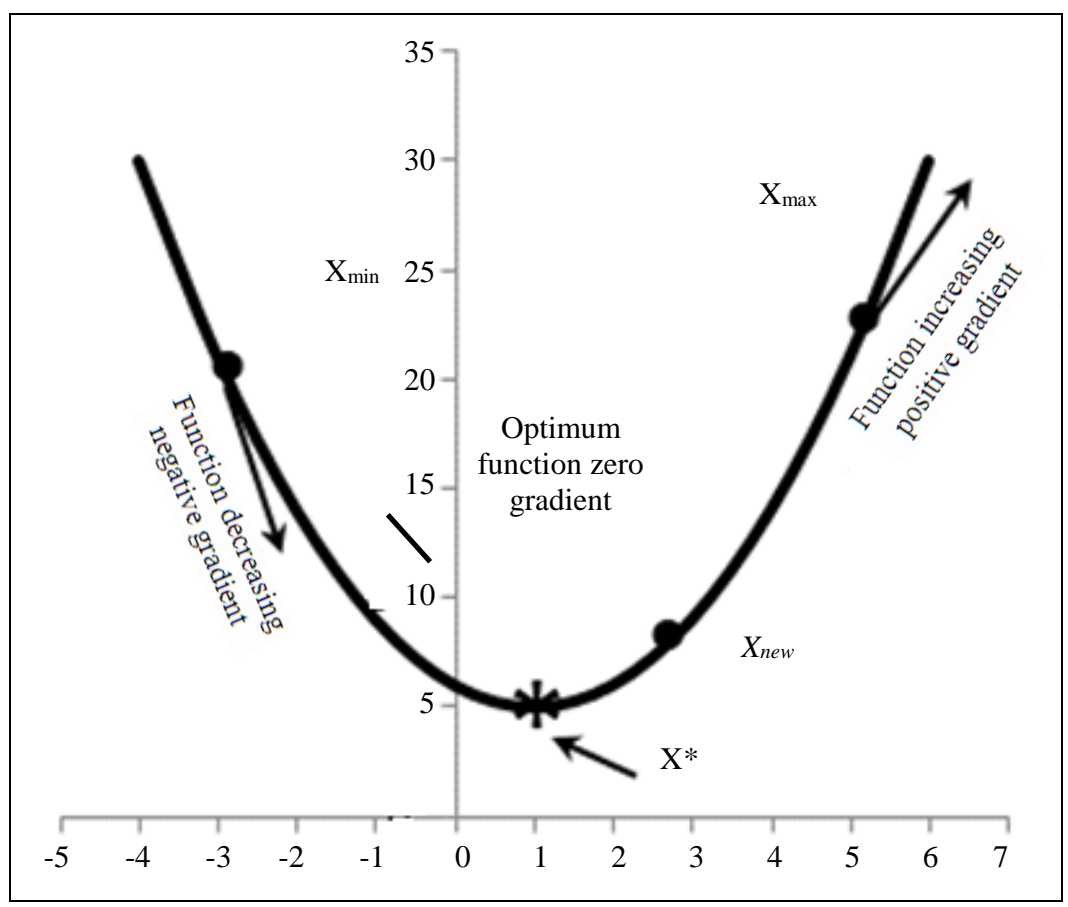

(a)

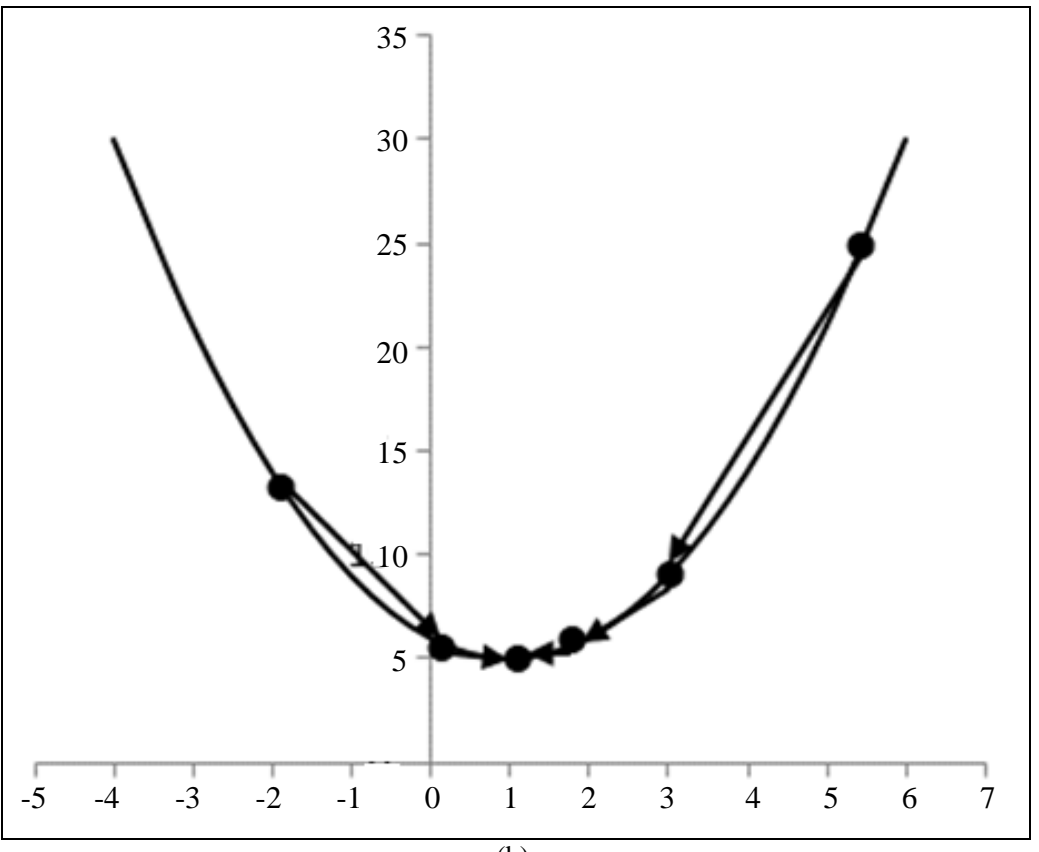

(b)

Fig. 1: Describe of CFDSM method 


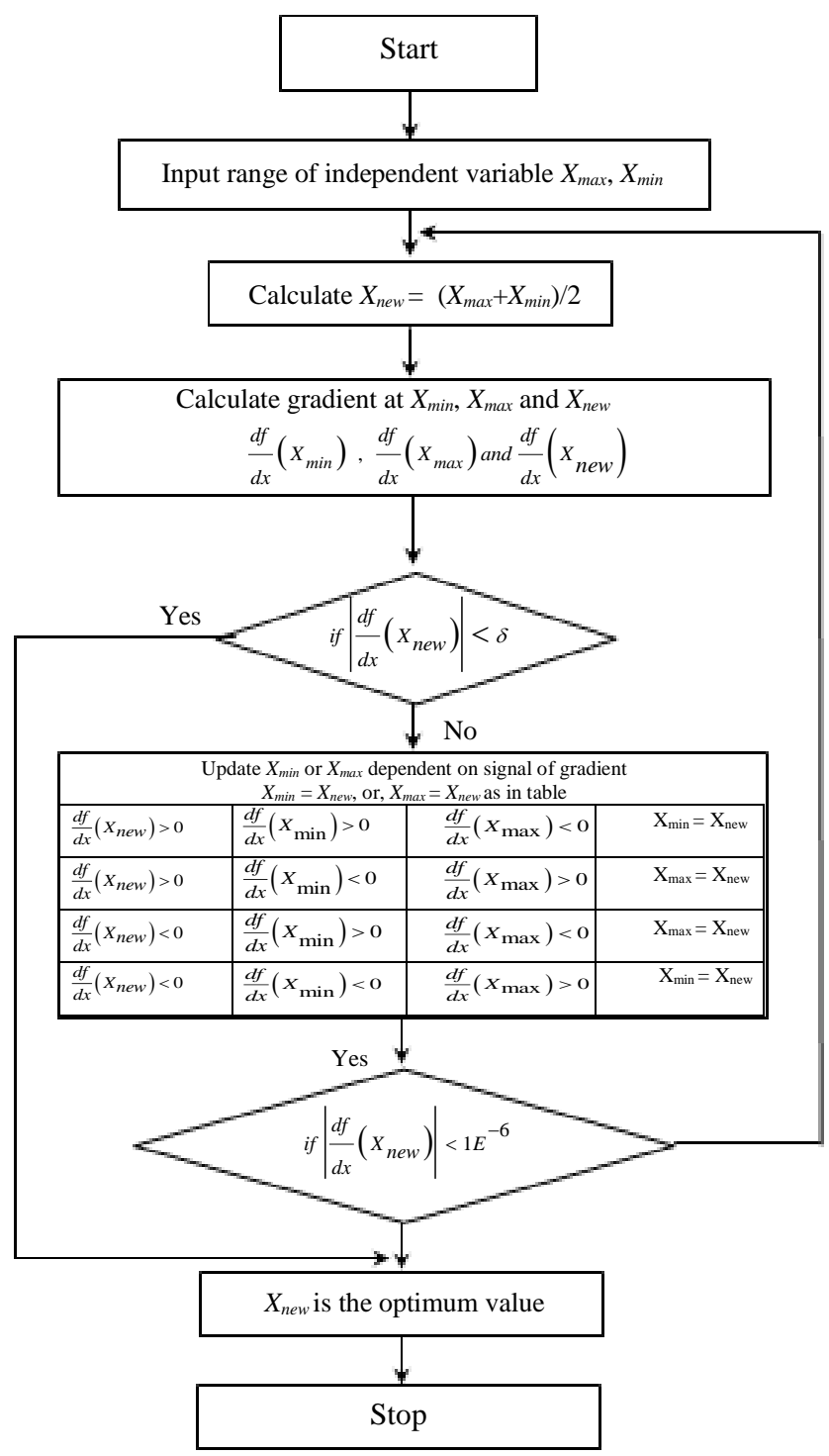

Fig. 2: Flow chart of this method

\section{Results}

Three case study will be taken in this paper to show the validity of this method (CFDSM) as:

\section{Case 1 (Two Variable - Linear First Derivative)}

This method also can be applied for the function has two independent variables or more than. Figure 3 show function-two variables has optimum value, where it have the form:

$$
z=(x-1)^{2}+(y-1)^{2}
$$

It has optimum value at $\left(x^{*}=1, y^{*}=1\right)$ and the minimum value is $z=0$. The gradient with respect to $x$ and $y$ of this function can be written as: $\frac{\partial f}{\partial x}=2(x-1)$

and:

$\frac{\partial f}{\partial y}=2(y-1)$

By solving the above two Equations after zeroes, the optimum value can be known. The Equation 4 is a linear function of $x$, it is independent on $y$. Also, the Equation 5 is a linear function of $y$, it is independent of $x$. Therefore easy solution of this function can be done. The optimum value of $x$ can be found as in above case given by Equation (1). Figure $2 \mathrm{~b}$ show an optimum value of $x$ will be constant along all range of another variable $(y)$. The optimum value 
of other variable $(y)$ can be found as $x$ and Fig. 3b show's it remains constant along all range of $x$.

\section{Case 2 (Two Variable - Nonlinear First Derivative)}

In this case a non-linear function $\left[z=x^{*} \exp \left(-x^{\wedge} 2-\right.\right.$ $\left.\left.y^{\wedge} 2\right)\right]$ in range of independent vairable $(0<x<2$ and $-2<y<2)$, which has optimum value (maximum) at $x^{*}=0.707$ and $y^{*}$ $=0$ as shown in Fig. 4 . In this case the ingredients are:

$\frac{\partial f}{\partial x}=\frac{1}{\exp \left(x^{2}+y^{2}\right)}-\frac{2 * x^{2}}{\exp \left(x^{2}+y^{2}\right)}$

$\frac{\partial f}{\partial y}=-\frac{2 * x * y}{\exp \left(x^{2}+y^{2}\right)}$

Also, update the maximum and minimum range of variable $x$ is calculated as explained in the flow chart shown in Fig. 2. But hear Equation 6 has other variable (y). In update and search for optimum value using Equation 6, the variable $y$ must take constant and it can take any value located in $y$-range. Figure $4 \mathrm{~b}$ it was $y_{\min }$ $\left(y_{\min }=-2\right)$ for only first iteration. In update maximum and minimum range of variable $y$ by using Equation 7, variable $x$ is taken as optimum value $\left(x_{n e w}\right)$ was updated by using Equation 6 in last update. Then, for all next iteration the others variable ( $x$ or $y$ ) using in Equation 6 and 7 are must be taken as ( $x_{\text {new }}$ and $y_{\text {new }}$ respectively). This procedure repeated till summation absolute value of gradient respect to $x$ and $y$ less small value $\left[\left|\frac{\partial f}{\partial x}\right|+\left|\frac{\partial f}{\partial y}\right|\right]_{\left(x_{n e w}, y_{n e w}\right)}<\delta$. Then the optimum value of two variables is got (Table 2).

\section{Case 3 (Compression with Others Optimization Method)}

In this case, a comparison results evaluated by this method with a genetic method. Equation 8, nonlinear two variables was chosen in this case:

$$
\begin{array}{rl}
z=3(1-\mathrm{x})^{2} & * \exp \left(-\left(\mathrm{x}^{2}\right)-(\mathrm{y}+1)^{2}\right)-10 \\
& *\left(\frac{\mathrm{x}}{5}-\mathrm{x}^{3}-\mathrm{y}^{5}\right) * \exp \left(-\mathrm{x}^{2}-\mathrm{y}^{2}\right)-\frac{1}{3} \\
& * \exp \left(-(\mathrm{x}+1)^{2}-\mathrm{y}^{2}\right)
\end{array}
$$

The Table 3 show the results calculated by two methods CFDSM and genetic for rang $(-2<x<2$ and $-2<y<2)$.

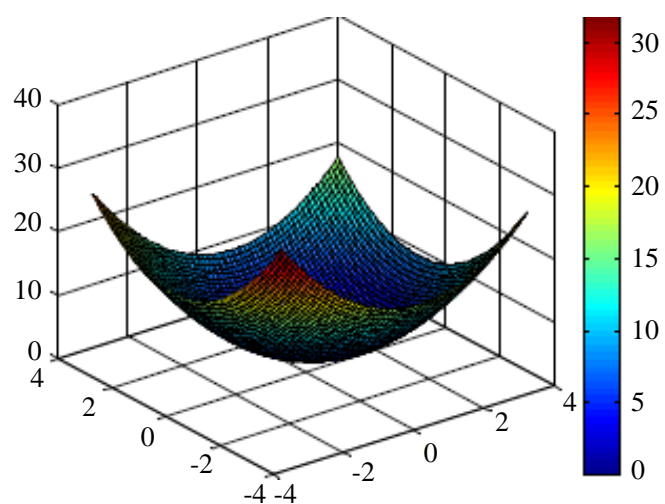

(a)

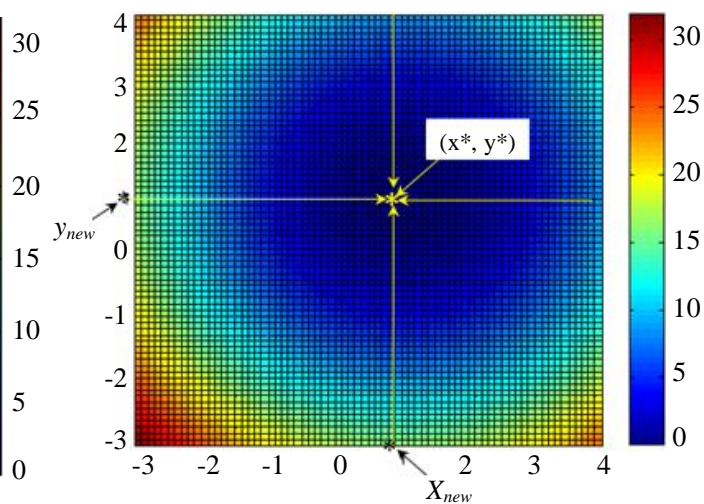

(b)

Fig. 3: The surface of Equation 3

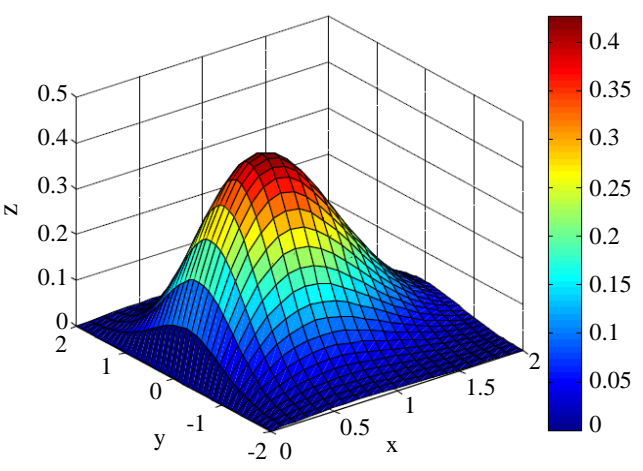

(a)

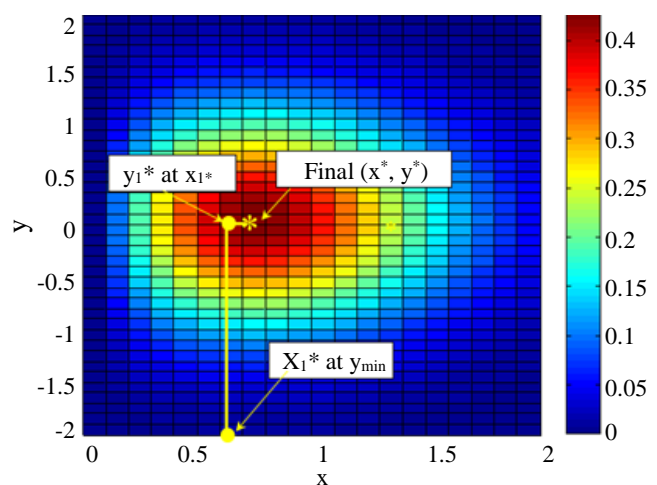

(b)

Fig. 4: The surface of equation $z=x^{*} \exp \left(-x^{\wedge} 2-y^{\wedge} 2\right)$ 
The Fig. 5 show surface of function that explained by Equation 8. This method (CFDSM) is very sensitive to the chosen range, where if the given range is chosen, we will get one point. But when we divide the range into several parts (divide the range into 9 parts) we will get the values in the Table 3 .

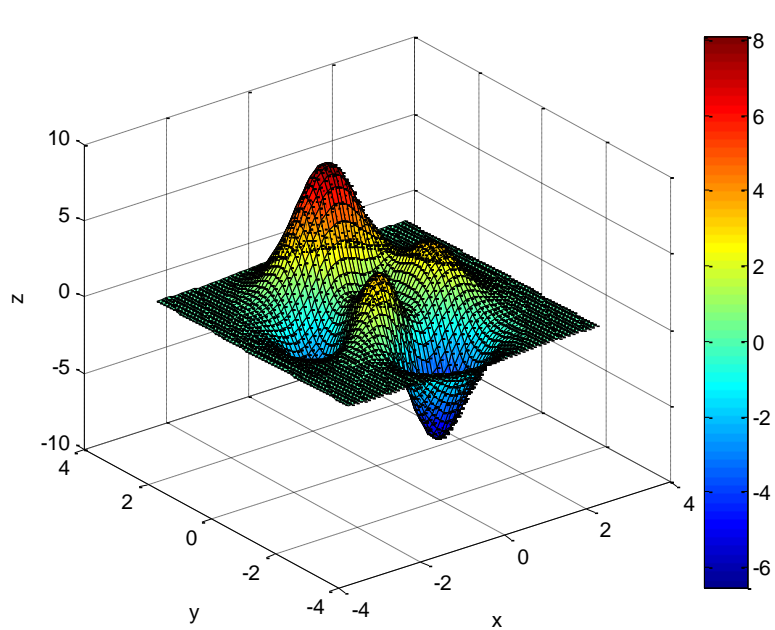

(a)

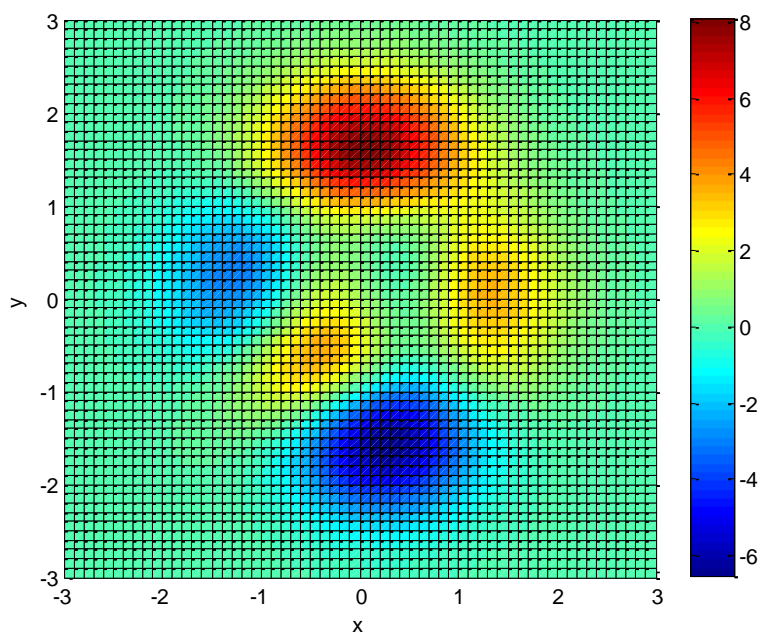

(b)

Fig. 5: (a) the surface of Equation 8 in 3-dimensions (b) the minimum and maximum values of the Equation 8 in 2-dimensions

Table 2: Shows the iteration

\begin{tabular}{|c|c|c|c|c|c|}
\hline \multirow[b]{2}{*}{ Iteration } & \multicolumn{2}{|c|}{ calculation $\mathrm{x} *\left(x_{\text {new }}\right)$} & \multicolumn{3}{|c|}{ calculation $\mathrm{y}^{*}$} \\
\hline & $x_{n e w}$ & $y$ & Iteration & $\mathrm{x}$ & $y_{\text {new }}$ \\
\hline 1 & 1.00000 & -2 & 17 & 0.70710 & 0.5 \\
\hline 2 & 0.50000 & -2 & 18 & 0.70710 & -0.25 \\
\hline 3 & 0.75000 & -2 & 19 & 0.70710 & 0.125 \\
\hline 4 & 0.62500 & -2 & 20 & 0.70710 & -0.0625 \\
\hline 5 & 0.68750 & -2 & 21 & 0.70710 & 0.0313 \\
\hline 6 & 0.71880 & -2 & 22 & 0.70710 & -0.0156 \\
\hline 7 & 0.70310 & -2 & 23 & 0.70710 & 0.0078 \\
\hline 8 & 0.71090 & -2 & 24 & 0.70710 & -0.0039 \\
\hline 9 & 0.70700 & -2 & 25 & 0.70710 & 0.002 \\
\hline 10 & 0.70900 & -2 & 26 & 0.70710 & $-9.77 \mathrm{E}-04$ \\
\hline 11 & 0.70800 & -2 & 27 & 0.70710 & $4.88 \mathrm{E}-04$ \\
\hline 12 & 0.70750 & -2 & 28 & 0.70710 & $-2.44 \mathrm{E}-04$ \\
\hline 13 & 0.70730 & -2 & 29 & 0.70710 & $1.22 \mathrm{E}-04$ \\
\hline 14 & 0.70720 & -2 & 30 & 0.70710 & $-6.10 \mathrm{E}-05$ \\
\hline 15 & 0.70710 & -2 & 31 & 0.70710 & $3.05 \mathrm{E}-05$ \\
\hline 16 & 0.70710 & -2 & 32 & 0.70710 & $-1.53 \mathrm{E}-05$ \\
\hline \multirow[t]{9}{*}{$\frac{\partial f}{\partial x}=2.9980 \mathrm{e}-8$} & 33 & 0.70710 & 7.63E-06 & & \\
\hline & 34 & 0.70710 & $-3.81 \mathrm{E}-06$ & & \\
\hline & 35 & 0.70710 & $1.91 \mathrm{E}-06$ & & \\
\hline & 36 & 0.70710 & $-9.54 \mathrm{E}-07$ & & \\
\hline & 37 & 0.70710 & 4.77E-07 & & \\
\hline & 38 & 0.70710 & $-2.38 \mathrm{E}-07$ & & \\
\hline & 39 & 0.70710 & 1.19E-07 & & \\
\hline & 40 & 0.70710 & $-5.96 \mathrm{E}-08$ & & \\
\hline & $\frac{\partial f}{\partial y}=5$ & & & & \\
\hline
\end{tabular}


Table 3: Compassion between CFDSM and genetic method

\begin{tabular}{|c|c|c|c|c|c|}
\hline \multicolumn{3}{|l|}{ CFDSM } & \multicolumn{3}{|l|}{ Genetic } \\
\hline $\mathrm{X}^{*}$ & $\mathrm{Y}^{*}$ & $\mathrm{Z}$ & $\mathrm{X}^{*}$ & $\mathrm{Y}^{*}$ & $\mathrm{Z}$ \\
\hline-1.3474 & 0.2045 & -3.0498 & -1.3434 & 0.2041 & -3.0497 \\
\hline-0.4600 & -0.6292 & 3.7766 & -0.4600 & -0.6293 & 3.7766 \\
\hline-0.2659 & 0.4667 & 0.7883 & -0.0093 & 1.5812 & 8.1062 \\
\hline-0.0093 & 1.5814 & 8.1062 & 0.2282 & -1.6255 & -6.5511 \\
\hline 0.2283 & -1.6255 & -6.5511 & & & \\
\hline 0.4163 & -0.3941 & 0.4085 & & & \\
\hline 0.2964 & 0.3202 & -0.0649 & & & \\
\hline .2857 & -0.0049 & 3.5925 & & & \\
\hline
\end{tabular}

\section{Conclusion}

Optimization methods are considered very important in engineering and industrial fields. Many of these methods depend on regression function for finding optimal values. In this research, a new method was adopted based on the change of the signal of the first derivative and three case study were tested to validate this method. The results show this method (CFDSM) can be used in optimization problems. The full range of design variables would be divided into several periods and the optimal values would be searched within these periods, when using the CFDSM method. The CFDSM method is an effective method of finding the optimal local and global values, for maximizing or minimizing objective function.

\section{Acknowledgement}

The authors express their deepest gratitude to the technical college of basrah and the college engineering of basrah.

\section{Author's Contributions}

All authors equally contributed in this work.

\section{Ethics}

This article is original and contains unpublished material. The corresponding author confirms that all of the other authors have read and approved the manuscript and there are no ethical issues involved.

\section{References}

Pardalos, P.M. and H.E. Romeijn, 2013. Handbook of Global Optimization. 1st Edn., Springer Science and Business Media, ISBN-10: 1461520258, pp: 880.

Rao, S.S., 1996. Engineering Optimization Theory and Practice. 1st Edn., John Wiley and Sons, Inc. ISBN-10: 0471550345, pp: 903.

Rustagi, J.S., 2014. Optimization Techniques in Statistics. 1st Edn., Elsevier, ISBN-10: 1483295710, pp: 359.

Stephen, B. and L. Vandenberghe, 2004. Convex Optimization. 1st Edn., Cambridge University Press, ISBN-10: 0521833787, ssspp: 716. 